\title{
Oral anticoagulation for cerebral ischemia of arterial origin High initial bleeding risk
}

\author{
M. Torn, MD; A. Algra, MD; and F.R. Rosendaal, MD
}

\begin{abstract}
Article abstract-Background: The use of oral anticoagulant therapy for the prevention of arterial thromboembolism in patients who have had ischemic stroke is controversial. Coumarins may increase the bleeding risk in patients with cerebral ischemia of arterial origin. Objectives: 1) To calculate incidence rates of bleeding and thromboembolic events in patients with noncardiac cerebral ischemia who were treated routinely in an anticoagulation clinic. 2) To assess which factors contribute to the occurrence of events. 3) To determine the optimal intensity of oral anticoagulant therapy in these patients. Methods: The authors studied all patients treated for noncardiac cerebral ischemia at the Leiden anticoagulation clinic between 1993 and 1998. Outcome events were major hemorrhage, major arterial thromboembolism, and death. Results: The authors observed 356 patients for 644 patient-years. The incidence of major hemorrhage was 3.9 per 100 patient-years (95\% CI, 2.5 to 5.7) and that of thromboembolism was 3.0 per 100 patient-years (95\% CI, 1.8 to 4.6). The incidence of hemorrhage varied with the duration of treatment (relative risk [RR] of the first versus the second half-year, 3.8 ; $95 \% \mathrm{CI}, 1.9$ to 7.6 ), age (RR for age $>65$ years, $3.7 ; 95 \% \mathrm{CI}, 1.1$ to 12.3 ), and the intensity of oral anticoagulation (RR, 1.8 for each 0.5 international normalized ratio [INR] unit increase; 95\% CI, 1.5 to 2.3). The optimal intensity of oral anticoagulant therapy was 2.5 to $3.5 \mathrm{INR}$; the best target value was 3.0 INR. Conclusion. The risk of hemorrhage with anticoagulant therapy is high in patients with ischemic stroke of arterial origin but is mainly confined to early use and elderly patients.
\end{abstract}

NEUROLOGY 2001;57·1993-1999

Patients with atrial fibrillation ${ }^{1}$ or myocardial infarction ${ }^{24}$ benefit from treatment with coumarins; however, treatment introduces a moderate annual bleeding risk of $2 \%$ to $3 \%$. A study of coumarins in patients with atrial fibrillation and recent nondisabling cerebral ischemia showed a $65 \%$ reduction of recurrent strokes with an accompanying annual bleeding risk of less than $3 \%$ percent. ${ }^{5}$ These results suggest that oral anticoagulation may prevent stroke in a wider range of indications.

To investigate whether coumarins also could improve the outcome for patients with stroke of noncardiac origin, the Stroke Prevention in Reversible Ischemia Trial (SPIRIT) was designed. SPIRIT was an open, multicenter clinical trial in which patients with cerebral ischemia of presumed arterial origin were randomized between low-dose aspirin $(30 \mathrm{mg}$ daily) and full-dose anticoagulation (international normalized ratio [INR], 3.0 to 4.5$).{ }^{6}$ The trial was prematurely terminated after the first interim analysis revealed an excessive rate of bleeding complications (7\% per year) in patients randomized to oral anticoagulation. Subsequent analyses revealed differences between patients that might help to explain the observed risks; high INR levels as well as the presence of leukoaraiosis appeared to be related to the bleeding risk in particular. ${ }^{67}$

We thought that more data were needed to judge the effect of coumarins in patients with cerebral ischemia of noncardiac origin. Therefore, we decided to evaluate the anticoagulant therapy of patients with cerebral ischemia of arterial origin who were treated routinely in the Leiden anticoagulation clinic. With the use of our extensive database, we calculated incidence rates of bleeding and thromboembolic events and searched for factors that contributed to the occurrence of events.

Furthermore, we determined the optimal intensity of oral anticoagulation, defined as the INR level that offers the best balance between prevention of thromboembolism and occurrence of bleeding complications. The position of this balance would tell us whether lowering the bleeding risk by a downshift of the target level, as suggested by the SPIRIT investigators, is outweighed by increased thromboembolic events.

Methods. Oral anticoagulation clinics. In the Netherlands, regional anticoagulation clinics monitor all patients on oral anticoagulant. ${ }^{8}$ Patients visit the anticoagulation

From the Departments of Hematology (Drs Torn and Rosendaal) and Clinical Epıdemiology (Dr Rosendaal), Leiden Unıversity Medical Center, and Department of Neurology (Dr Algra) and Julius Center for Patient Oriented Research (Dr Algra), University Medical Center Utrecht, the Netherlands Supported by grants from the Dutch Thrombosis Foundation (No 94001), the Prevention Fund (no 28-2542), and The Netherlands Heart Foundation (no 96 114)

Recerved May 21, 2001. Accepted in final form August 17, 2001

Address correspondence and reprint requests to Dr F R Rosendaal, Department of Clmical Epıdemiology, Leiden University Medical Center, C9-P PO Box 9600,2300 RC Leiden, the Netherlands, e-mail fr rosendaal@lumc nl 
clinic every 1 to 6 weeks (average interval, 3 weeks) or are visited at home. During each visit, a nurse takes a short medical history with special attention to bleeding and thromboembolic events, changes in medication, intercurrent diseases, and hospital admissions. An antecubital blood sample is taken and prothrombin time is measured. Prothrombin times are expressed in INR, the international standard for assessing intensity of oral anticoagulation. ${ }^{9}$ Until autumn 1996, the target range for patients receiving anticoagulant therapy because of a prior stroke was 3.0 to 4.5 INR. Afterward, it was lowered and set at 2.5 to 3.5 INR.

Pattents. Because we sought to study anticoagulated patients with cerebral ischemia of noncardiac origin, we searched among the population of the Leiden anticoagulation clinic for patients who met this criterion. We included patients without cardiac embolic sources (i.e., without atrial fibrillation or cardiac thrombus) who were treated for cerebral ischemia between January 1, 1994, and January, 1 1998. Because antiplatelet agents, rather than coumarins, are the standard treatment for ischemic stroke in the Netherlands, the selected patients may not be representative of the general population of Dutch stroke survivors.

To determine whether registration of the anticoagulation clinic was reliable in distinguishing between patients with and without cardiac embolic sources, we took a random sample of 20 patients and evaluated their full medical charts. In 16 patients, no cardiac embolic source could be demonstrated, 3 had atrial fibrillation, and 1 had a cardiac thrombus on ultrasound.

Data collection. We included the following information from the computerized records: date of the index ischemic cerebral event and start of anticoagulant treatment, duration of treatment, date of birth, sex, dates and results of all prothrombin times, hospital admissions, and death. These data are at all times routinely collected by the anticoagulation clinic.

To register events on follow-up, we additionally collected discharge letters for all hospital admissions during the period of the study along with relevant results of laboratory tests, radiographs, CT scans, and autopsy reports. When patients died outside the hospital, we contacted their general practitioners about the cause of death.

Outcome events. Primary outcome events were major hemorrhages (intracranial, spinal, and extracranial) and arterial thromboembolic events (ischemic stroke, myocardial infarction, and peripheral embolism). Death (vascular and nonvascular) was considered a secondary outcome event.

Intracranial and spinal hemorrhage was defined as a neurologic deficit of sudden or subacute onset, confirmed by surgery, autopsy, or CT scan. Major extracranial hemorrhage was defined as acute blood loss, outward or inward, leading to death or to hospital admission for observation or treatment. Admission for diagnostic purposes only was not considered major extracranial hemorrhage, nor was a bleeding event that occurred while the patient was admitted. The diagnosis of ischemic stroke required a neurologic deficit of acute onset, proven by autopsy or CT scan. Myocardial infarction was defined by two of the following: history of chest discomfort, typical rise of specific cardiac enzymes, or the development of new $Q$ waves on EKG. Peripheral embolism was defined as sud- den peripheral ischemia, proven by duplex scanning, angiography, surgery, or autopsy. Death from vascular diseases included sudden death (reliable observation of the time between onset of symptoms and death or the patient being found dead) or death from myocardial infarction, congestive heart failure, peripheral embolism, stroke, pulmonary embolism, any bleed, or other vascular causes.

An expert panel, composed of a cardiologist, neurologist, internist, and clinical epidemiologist, classified all events according to these definitions. The panel members were at all times blinded for the intensity of anticoagulation at the time of the event.

Optımal intensity of anticoagulation. The optimal intensity of anticoagulant therapy was defined as the INR range with the lowest incidence of untoward events. Intensity-specific incidence rates were calculated as the ratio of the number of events that took place at a certain INR level and the number of patient-years that this intensity level had been achieved by the total patient population. INR-specific patient-years were computed using a method that converts patient-time into INR time by assuming a linear relationship between consecutive INR measurements. ${ }^{10}$ All days with the same achieved intensity level are summed and grouped in intervals of $0.5 \mathrm{INR}$. The INR at the time of an outcome event was obtained from the hospital records. If the INR was not measured on hospital admission or if the test result could not be retrieved, we used the last INR measurement at the anticoagulation clinic within 8 days before the event. If no suitable INR was available, the event was disregarded for the calculation of incidence rates.

Data analysis. Incidence rates and their 95\% CI were derived by standard calculations, based on the assumption of a Poisson distribution of the number of events. A Cox proportional hazards model was used to compare the incidence of outcome events between groups. Differences between groups were expressed as a hazard ratio (HR), which is synonym to the relative risk.

The mortality data of our study and the SPIRIT trial were standardized by age to allow comparison between the studies. Standardization was performed by 5 -year age categories, with the pooled age distribution as weights.

Results. Patients. During the follow-up period, 381 patients were anticoagulated because of prior cerebral ischemia. Five patients had participated in the SPIRIT study and were therefore excluded. Another 20 patients were excluded because they were known to have atrial fibrillation.

At the start of the study period, January 1, 1994, 132 patients were treated for cerebral ischemia that had occurred before that date, whereas 224 patients had an ischemic cerebral event between January 1, 1994, and January 1,1998 , and were subsequently treated with coumarins. In 180 patients, treatment was discontinued: 63 patients died, 11 stopped after a bleeding event, 28 moved out of the region to another anticoagulation clinic, 48 switched to aspirin, and 30 patients discontinued treatment for other reasons.

The total observation time was 644 patient-years, the mean observation per patient was 1.8 years. The mean age of the patients was 69 years, ranging from 24 to 93 . Fortyfive percent were women. During the 4 years of follow-up, 
Table 1 Outcome events

\begin{tabular}{|c|c|c|}
\hline Outcome & $\begin{array}{c}\text { Events } \\
(n=107)\end{array}$ & $\begin{array}{c}\text { Incidence* }(95 \% \mathrm{CI}) \\
644 \text { patient-years }\end{array}$ \\
\hline \multicolumn{3}{|l|}{ Major bleeding } \\
\hline \multicolumn{3}{|l|}{ Fatal } \\
\hline Intracranial & 7 & 1.1 \\
\hline Gastrointestinal & 2 & 0.3 \\
\hline \multicolumn{3}{|l|}{ Nonfatal } \\
\hline Intracranial & 3 & 0.5 \\
\hline Gastrointestinal & 7 & 1.1 \\
\hline Urogenital & 2 & 0.3 \\
\hline Other & 4 & 0.6 \\
\hline Total & 25 & $3.9(2.5-5.7)$ \\
\hline \multicolumn{3}{|l|}{ Major thromboembolism } \\
\hline \multicolumn{3}{|l|}{ Fatal } \\
\hline Ischemic stroke & 2 & 0.3 \\
\hline Myocardial infarction & 2 & 0.3 \\
\hline \multicolumn{3}{|l|}{ Nonfatal } \\
\hline Ischemic stroke & 10 & 1.6 \\
\hline Myocardial infarction & 5 & 0.8 \\
\hline Total & 19 & $3.0(1.8-4.6)$ \\
\hline \multicolumn{3}{|l|}{ Death } \\
\hline Vascular & 37 & 5.7 \\
\hline Nonvascular & 25 & 3.9 \\
\hline Unknown cause & 1 & 0.2 \\
\hline Total & 63 & $9.8(7.5-12.4)$ \\
\hline
\end{tabular}

* The number of patient-years is the maximum follow-up period, based on the secondary outcome event "death of all causes." Because follow-up ended when the endpoint of interest occurred, the number of patient-years differs slightly between endpoints. The incidence rate is expressed per 100 patientyears.

217 hospital admissions in 139 patients were reported. Sufficient clinical information was available in $98 \%$ of the admissions.

Anticoagulant control. During the follow-up period, 10,628 INR measurements were routinely performed at the anticoagulation clinic. The average period between two measurements was 3 weeks. In the initial 6 months of treatment, $55 \%$ of the follow-up time was spent within the target range. Approximately 68\% of the time was spent within this intensity range overall. Thirty patient-years were not allocated to an INR interval because the duration between two measurements exceeded 8 weeks. ${ }^{10}$

Incidences of primary and secondary outcome events. Table 1 presents an overview of all outcome events. Sufficient clinical data were available to classify all strokes as either hemorrhagic or ischemic. Twenty-five patients had a major bleeding event (3.9 per 100 patient-years; $95 \% \mathrm{CI}$, 2.5 to 5.7 ) of which nine were fatal (1.4 per 100 patientyears; $95 \% \mathrm{CI} ; 0.6$ to 2.5 ): seven patients had a fatal intracranial hemorrhage and two patients died because of massive gastrointestinal blood loss. Nonfatal bleeding events were intracranial (3), gastrointestinal (7), urogenital (2), muscular (1), retroperitoneal (1), joint (1) and nose

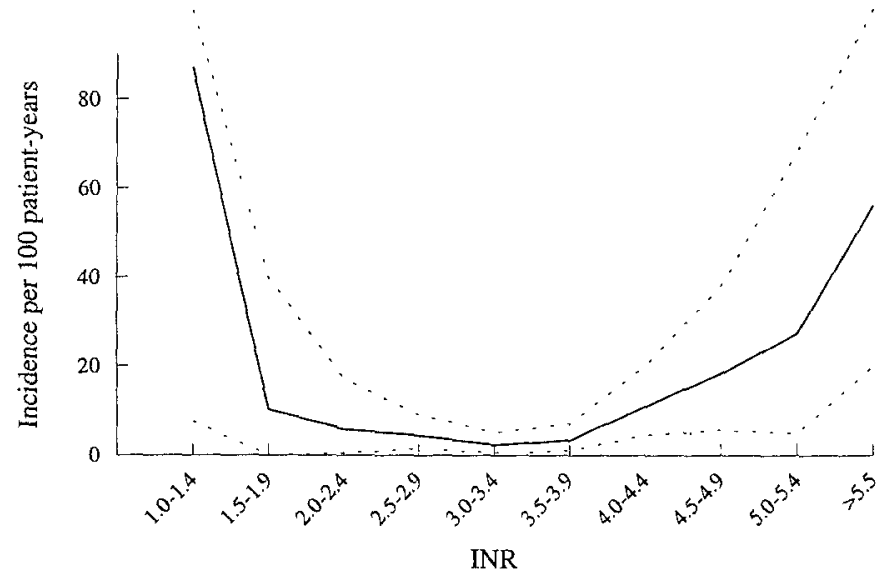

Figure 1. International normalized ratio (INR)-specific incidence rates of all primary outcome events. The corresponding numbers are presented in table 2. The dotted lines represent the $95 \% \mathrm{CI}$.

bleeds (1). The incidence of fatal and nonfatal intracranial hemorrhage combined was 1.6 per 100 patient-years $(95 \% \mathrm{CI}$, 0.7 to 2.7 ).

Nineteen patients had thromboembolic events (3.0 per 100 patient-years; 95\% CI, 1.8 to 4.6 ). Two ischemic strokes and two myocardial infarctions were fatal, yielding an incidence rate for fatal thromboembolism of 0.6 per 100 patient-years (95\% CI, 0.2 to 1.4). Nonfatal thromboembolic events were ischemic stroke (10) and myocardial infarction (5).

A total of 63 patients died during follow-up (9.8 per 100 patient-years; $95 \%$ CI, 7.5 to 12.4 ). The cause of death could be retrieved for all but one patient. In 37 patients, the cause of death was classified as vascular (5.7 per 100 patient-years; $95 \% \mathrm{CI}, 4.0$ to 7.8 ). Thirteen patients died because of primary outcome events. The causes of death for the remaining 24 patients were cardiac, presumed myocardial infarction (1), the sequel of a primary cardiac event (2), or congestive heart failure (2); cerebral, acute onset of neurologic deficit without hospital admission (5) or slow deterioration after multiple infarctions (3); vascular, pulmonary embolism (1) and aortic dissection (1); and sudden death (9).

Optimal intensity of anticoagulation. We were able to retrieve INR values at the time of the event for 41 of 44 patients who had primary outcome events. Thirty-six prothrombin times were measured directly after the event whereas five INR values were obtained from the records of the anticoagulation clinic and had been determined within 8 days before the event.

Figure 1 shows the incidence rates of all combined untoward events and their 95\% CI per INR interval. The lowest incidence (2.3 per 100 patient-years; $95 \% \mathrm{CI}, 0.6$ to 5.1) was found between 3.0 and $3.5 \mathrm{INR}$; the incidence rates rose steeply below 2.0 and over 4.5 INR. The position of the curve remained basically unaltered if the 24 patients who died from vascular causes outside the hospital also were taken into account.

Table 2 presents INR-specific incidence rates of all major bleeding and thromboembolic events. The risk ratio for hemorrhage was 1.8 per $0.5 \mathrm{INR}$ unit increase (95\% CI, 1.5 to 2.3$)$ 
Table 2 Incidence rates of untoward events per INR interval

\begin{tabular}{|c|c|c|c|c|c|c|c|}
\hline $\begin{array}{l}\text { INR } \\
\text { interval }\end{array}$ & $\begin{array}{c}\text { INR } \\
\text { years } \\
(\mathrm{n}=614)\end{array}$ & $\begin{array}{c}\text { Major } \\
\text { hemorrhage } \\
(\mathbf{n}=22)\end{array}$ & $\begin{array}{c}\text { Major } \\
\text { thromboembolism } \\
(\mathrm{n}=19)\end{array}$ & $\begin{array}{l}\text { All events } \\
(\mathrm{n}=41)\end{array}$ & $\begin{array}{c}\text { Incidence } \\
\text { hemorrhage* }\end{array}$ & $\begin{array}{c}\text { Incidence } \\
\text { thromboembolism* }\end{array}$ & $\begin{array}{l}\text { Incidence } \\
\text { overall* }\end{array}$ \\
\hline $1.0-1.4$ & 2.3 & 0 & 2 & 2 & - & 86.9 & 86.9 \\
\hline $1.5-1.9$ & 9.8 & 0 & 1 & 1 & - & 10.2 & 10.2 \\
\hline $2.0-2.4$ & 35.2 & 1 & 1 & 2 & 2.8 & 2.8 & 5.7 \\
\hline $2.5-2.9$ & 112.5 & 1 & 4 & 5 & 0.9 & 3.6 & 4.4 \\
\hline $30-3.4$ & 177.2 & 3 & 1 & 4 & 1.7 & 0.6 & 2.3 \\
\hline $3.5-3.9$ & 154.2 & 4 & 1 & 5 & 2.6 & 0.6 & 3.3 \\
\hline $4.0-4.4$ & 737 & 3 & 5 & 8 & 4.1 & 6.8 & 10.9 \\
\hline $4.5-4.9$ & 27.4 & 4 & 1 & 5 & 14.6 & 3.6 & 18.3 \\
\hline $5.0-5.5$ & 11.0 & 1 & 2 & 3 & 9.1 & 18.2 & 27.3 \\
\hline$>5.5$ & 10.6 & 5 & 1 & 6 & 47.2 & 9.4 & 56.6 \\
\hline
\end{tabular}

+ Incidence per 100 patient-years.

$\mathrm{INR}=$ international normalized ratio.

Risk factor analyses. Age. To assess the effect of age on the occurrence of outcome events in our study, we drew Kaplan-Meier curves for all primary outcome events (figure 2A) and overall survival (figure 2B). Advanced age had a considerable effect on the occurrence of primary outcome events (HR for age $>65$ years, $1.7 ; 95 \% \mathrm{CI}, 0.8$ to 3.4 ) and an even more profound effect on survival ( $\mathrm{HR}, 2.4 ; 95 \% \mathrm{CI}$, 1.3 to 4.7 ).

Further analyses of the relationship between age and primary outcome events revealed a different effect of age on bleeding and on thromboembolism. Advanced age was a significant risk factor for the occurrence of major bleeding (HR, 3.7; 95\% CI, 1.1 to 12.3 ) but did not increase the risk of thromboembolism (HR, 0.7; 95\% CI, 0.3 to 1.8).

Duration of treatment. In clinical trials such as SPIRIT, most patients randomized to oral anticoagulation are new to coumarins therapy. In our study, however, a number of patients had already been treated with coumarins for years before follow-up started and, therefore, we could assess long-term effects. We divided into five categories the time interval between the cerebral ischemic event that led to prescription of coumarin and the start of follow-up in our study, with the shortest time interval (up to 3 months) as the reference category. The hazard ratio for all primary outcome events was 0.94 (95\% CI, 0.13 to 6.95) for patients who entered the study between 3 and 6 months after the index event. After the first 6 months, the hazard ratios decreased to 0.28 in the second half-year (95\% CI, 0.04 to 2.07 ), to 0.20 after 1 to 3 years (95\% CI, 0.03 to 1.48 ) and to 0.55 after more than 3 years $(95 \% \mathrm{CI}$, 0.28 to 1.10 ), indicating that the risk of untoward events is highest in the first 6 months after cerebral ischemia.

To further quantify these differences, we analyzed starters and long-term users separately (table 3 ). The 228 starters (276 patient-years) were new to oral anticoagulation or used coumarins for less than 6 months when follow-up started, whereas the 128 long-term users (368 patient-years) had already been treated with oral anticoagulants for more than 6 months after an episode of cerebral ischemia. Elderly patients ( $>65$ years of age) were equally represented in both groups (69.3\% versus $71.9 \%$ ).
Twenty-nine primary outcome events were counted in the starters group. Nine patients had cerebral hemorrhages (3.3 per 100 patient-years; $95 \%$ CI, 1.5 to 5.8 ), of which six were fatal. The overall incidence of major bleeding events was 6.2 per 100 patient-years (95\% CI, 3.6 to 9.6). Starters also had a high incidence of thromboembolic events. The incidence rate of all combined thromboembolic endpoints was 4.5 per 100 patient-years (95\% CI, 2.3 to 7.4). Among the long-term users, 15 primary outcome events occurred. There was only one cerebral hemorrhage among the eight bleeding events ( 2.2 per 100 patient-years; $95 \% \mathrm{CI}, 0.9$ to 4.0). The overall incidence of thromboembolic events was less than half of that in the starters group (1.9 per 100 patient-years; $95 \%$ CI, 0.8 to 3.7 ).

Therefore, starters had a 2.6-fold higher risk of untoward events than did long-term users (95\% CI, 2.5 to 2.6 ), and that risk was composed both of a higher bleeding risk (RR, 2.8; 95\% CI, 2.6 to 3.0 ) and a higher thromboembolic risk (RR, 2.4; 95\% CI, 2.1 to 2.6 ).

Finally, we studied the occurrence of primary outcome events in the first year of treatment after the episode of cerebral ischemia by comparing the incidence rates in the initial 6 months of anticoagulant therapy with the rates in the second half-year. Results are presented in table 4. During the first 6 months of coumarin therapy, 10 hemorrhages (7 intracranial), four myocardial infarctions, and four ischemic strokes occurred in 83 patient-years, yielding incidence rates of 12.0 per 100 patient-years (95\% CI, 5.6 to 20.9) for major hemorrhage and of 9.7 per 100 patientyears (95\% CI, 4.1 to 17.8 ) for thromboembolism. In the second half-year (62 patient-years) two extracranial hemorrhages (3.2 per 100 patient-years; $95 \%$ CI, 0.3 to 9.4 ) and three arterial thromboses (4.9 per 100 patient-years; $95 \%$ CI, 0.9 to 12.1 ) were registered.

These numbers yield a RR of 2.7 (95\% CI, 2.4 to 3.1 ) in detriment of the first 6-month period for all primary outcome events and of 3.8 (95\% CI, 1.9 to 7.6 ) for hemorrhage only. The overall incidence rate of primary outcome events in the first year of anticoagulant treatment was 8.3 per 100 patient-years (95\% CI, 4.2 to 13.7 ) for all major hem- 


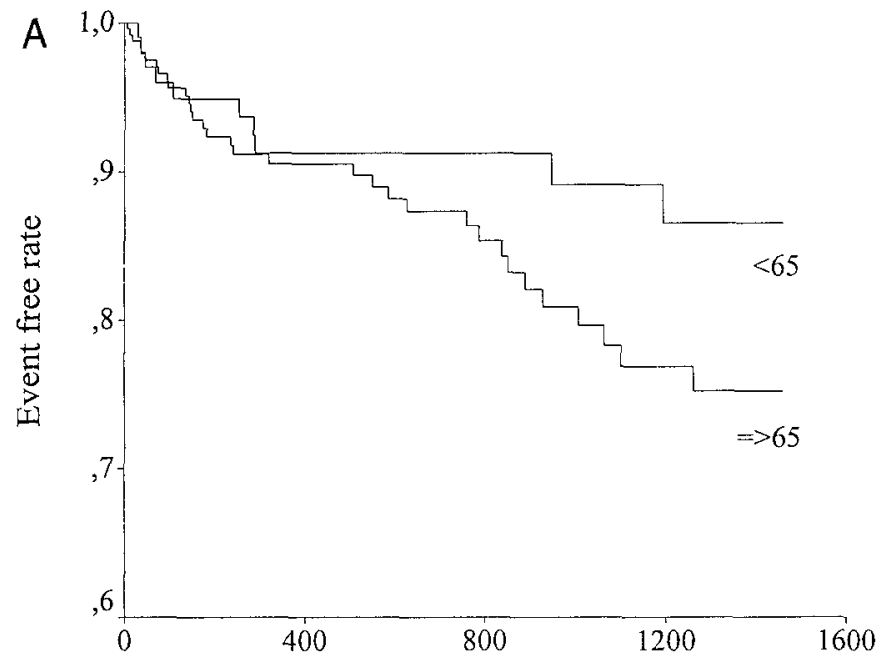

Time (days)

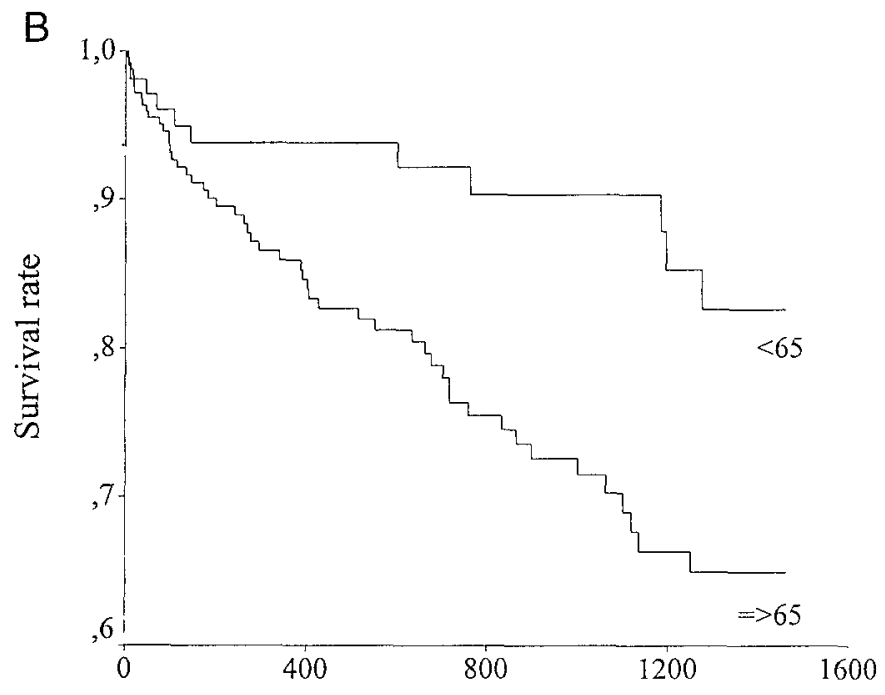

Time (days)

Figure 2. (A) Kaplan-Meier curve for all primary outcome events according to age older than and younger than 65 years. (B) Kaplan-Meier curve for overall survival according to age older than and younger than 65 years.

orrhage, 4.8 (95\% CI, 1.9 to 9.2 ) for intracranial bleeding, and 7.6 (95\% CI, 3.7 to 12.9 ) for arterial thromboembolism.

Discussion. To study the effect of coumarins in patients with cerebral ischemia of noncardiac origin, we performed an observational study among patients of a Dutch anticoagulation clinic. In these patients, we found an overall bleeding incidence of 3.9 per 100 treatment-years (95\% CI, 2.5 to 5.7 ), which is less than the annual bleeding risk in a previous randomized trial $(7.0 ; 95 \% \text { CI, } 5.4 \text { to } 9.3)^{6}$ but higher than the bleeding incidences in studies of other indications $(2 \% \text { to } 3 \%)^{1,3}$ Risk factor analyses revealed a major effect of age (HR for age $>65$ years, 3.7), duration of anticoagulant treatment after cerebral ischemic event (RR in the first 6 months, 3.8), and of the achieved intensity of oral anticoagulation (RR, 1.8 for each 0.5 INR unit increase) on the occurrence of bleeding events.

Differences between the outcomes of our study and the randomized trial (SPIRIT) may be because of distinct patient characteristics such as the age distribution of participants: $71 \%$ of our patients were older than 65 years ( 65 to 93 ; mean, 75 ) versus only $47 \%$ in SPIRIT. Part of the high mortality rate in our study can be explained by this difference. When standardized according to age, the mortality rate in our cohort declined from 9.8 to 8.2 per 100 patientyears, whereas that of spirit increased from 4.5 to 5.0 per 100 patient-years. The remaining surplus of secondary endpoints may be explained by a less favorable risk profile of the routinely treated patient group compared with the patients in the randomized trial.

We also found an association between age and risk of hemorrhage, a relationship that has already been described extensively in the literature. ${ }^{11,12}$ The increasing burden of comorbidity in the elderly may explain this finding. ${ }^{12,13}$

The incidence of thromboembolism in starters was more than twice as high as in long-term users of oral anticoagulant therapy. There are several possible explanations for the high incidence rate among starters. First, the intensity of oral anticoagulation is often unstable in the starting phase of anticoagulant treatment. In our study, $55 \%$ of the observation time during the first 6 months of treatment was spent in the target range compared with $70 \%$ thereafter. Because the risk of thromboembolism rises steeply if the achieved intensity level drops below 2.0 INR, more thromboembolic events are expected in the first months of therapy. Second, the risk of a recurrent event may be high during the first few months after cerebral ischemia. Even if anticoagulation has been adequate, the thromboembolic risk might have exceeded its preventive capacities.

We also found a considerable difference in bleeding rates between starters and long-term users. Long-term users, who received oral anticoagulation for at least 6 months before our study, had bleeding rates comparable with those in other studies; the annual rates of $0.5 \%$ for fatal and $2.2 \%$ for major hemorrhages are very similar to the results of a previous study on the bleeding risk for all patients of the Leiden anticoagulation clinic. ${ }^{14}$ Starters, however, had a nearly three times greater bleeding incidence (6.2 per 100 patient-years) than long-term users, mainly because of an excessive number of cerebral hemorrhages. The excess of cerebral bleeding events in starters clustered exclusively in the initial 6 months of coumarin therapy. When we considered the entire first year of treatment, the incidence of hemorrhage was 8.3 per 100 patient-years $(95 \% \mathrm{CI}$, 4.2 to 13.7 ), which corresponds very well with the SPIRIT trial, in which a bleeding incidence of 7.0 (95\% CI, 5.4 to 9.3 ) was found.

In general, the bleeding incidence among anticoagulated patients tends to be higher during the first 
Table 3 Outcome events: Starters vs long-term users

\begin{tabular}{|c|c|c|c|c|}
\hline \multirow[b]{2}{*}{ Outcome } & \multicolumn{2}{|c|}{ Starters (index event $\leq 6$ months) } & \multicolumn{2}{|c|}{ Long-term users (index event $>6$ months) } \\
\hline & $\begin{array}{c}\text { Events, } \\
\quad \mathrm{n}\end{array}$ & $\begin{array}{l}\text { Incidence }(95 \% \mathrm{CI}) \\
276 \text { patient-years }\end{array}$ & $\begin{array}{c}\text { Events, } \\
\mathrm{n}\end{array}$ & $\begin{array}{l}\text { Incidence* }(95 \% \mathrm{CI}) \\
368 \text { patient-years }\end{array}$ \\
\hline \multicolumn{5}{|l|}{ Major bleeding } \\
\hline \multicolumn{5}{|l|}{ Fatal } \\
\hline Intracranial & 6 & 2.2 & 1 & 0.3 \\
\hline Other & 1 & 0.4 & 1 & 0.3 \\
\hline \multicolumn{5}{|l|}{ Nonfatal } \\
\hline Intracranial & 3 & 1.1 & 0 & - \\
\hline Other & 7 & 2.6 & 6 & 1.6 \\
\hline Total & 17 & $6.2(3.6-9.6)$ & 8 & $2.2(0.9-4.0)$ \\
\hline \multicolumn{5}{|l|}{ Major thromboembolism } \\
\hline \multicolumn{5}{|l|}{ Fatal } \\
\hline Ischemic stroke & 2 & 0.7 & 0 & - \\
\hline Myocardial infarction & 1 & 0.4 & 1 & 0.3 \\
\hline \multicolumn{5}{|l|}{ Nonfatal } \\
\hline Ischemic stroke & 5 & 1.9 & 5 & 1.4 \\
\hline Myocardial infarction & 4 & 1.5 & 1 & 0.3 \\
\hline Total & 12 & $4.5(2.3-7.4)$ & 7 & $1.9(0.8-3.7)$ \\
\hline \multicolumn{5}{|l|}{ Death } \\
\hline Vascular & 23 & 8.3 & 14 & 3.8 \\
\hline Nonvascular & 12 & 4.3 & 13 & 3.5 \\
\hline Unknown cause & 0 & - & 1 & 0.3 \\
\hline Total & 35 & $12.7(8.8-17.3)$ & 28 & $7.6(5.0-10.8)$ \\
\hline
\end{tabular}

* The number of patient-years between parentheses is the maximum follow-up period, based on the secondary outcome event "death of all causes." Because follow-up ended when the endpoint of interest occurred, the number of patient-years differs slightly between endpoints. The incidence rate is expressed per 100 patient-years.

months of treatment. ${ }^{\text {I1 }}$ The instability of anticoagulation during the starting phase of oral anticoagulant therapy and also the increased vulnerability of patients with an existing (silent) bleeding site partly explain this phenomenon. Because the excess number of bleeding events in patients with cerebral ischemia of noncardiac origin exclusively concerned intracranial and not extracranial hemorrhage, it is

Table 4 Outcome events: Initial 6 months vs second 6 months of treatment

\begin{tabular}{|c|c|c|c|c|}
\hline \multirow[b]{2}{*}{ Outcome } & \multicolumn{2}{|c|}{$\begin{array}{c}\text { Initial } 6 \text { months of anticoagulant } \\
\text { treatment }\end{array}$} & \multicolumn{2}{|c|}{$\begin{array}{c}\text { Second } 6 \text { months of anticoagulant } \\
\text { treatment }\end{array}$} \\
\hline & $\begin{array}{c}\text { Events, } \\
\text { n }\end{array}$ & $\begin{array}{l}\text { Incidence* }(95 \% \mathrm{CI}) \\
83 \text { patient-years }\end{array}$ & $\begin{array}{c}\text { Events, } \\
n\end{array}$ & $\begin{array}{l}\text { Incidence* }(95 \% \mathrm{CI}) \\
62 \text { patient-years }\end{array}$ \\
\hline \multicolumn{5}{|l|}{ Major bleeding } \\
\hline Intracranial & 7 & 8.4 & 0 & 一 \\
\hline Other & 3 & 3.6 & 2 & 3.2 \\
\hline Total & 10 & $12.0(5.6-20.9)$ & 2 & $3.2(0.3-9.4)$ \\
\hline \multicolumn{5}{|l|}{ Major thromboembolism } \\
\hline Ischemic stroke & 4 & 4.9 & 3 & 4.9 \\
\hline Myocardial infarction & 4 & 4.8 & 0 & - \\
\hline Total & 8 & $9.7(4.1-17.8)$ & 3 & $4.9(0.9-12.1)$ \\
\hline
\end{tabular}

1998 NEUROLOGY 57 December (1 of 2) 2001 
reasonable to look for intracranial abnormalities that distinguish the patients at high risk from other patients. Several attempts have been made to identify these patients. ${ }^{6,7}$ Leukoaraiosis-a white matter abnormality on CT scan-is a potential risk factor. Leukoaraiosis often results from structural changes in small cerebral arteries and arterioles ${ }^{15}$ and might lead to increased fragility of the vessel wall and subsequent bleeding. ${ }^{16}$ However, different forms of leukoaraiosis exist and their precise role and meaning are not yet fully understood. Arterial hypertension was also identified as a risk factor for bleeding, ${ }^{11,16}$ but opposite results have been published as well. ${ }^{12,17}$ Further research will be necessary to identify patients with high bleeding risk to offer them a different treatment.

Many studies have already proven the value of determining optimal intensity of oral anticoagulation by using the actual achieved intensity of oral anticoagulation. ${ }^{17-19}$ The optimal intensity level of oral anticoagulation in this cohort was between 3.0 and 3.5 INR. Preferably, most treatment time should be spent in this range to minimize the occurrence of untoward events, whereas intensity levels below 2.0 and above 4.5 INR should be avoided. To reach these goals, a target level of 2.5 to 3.5 INR with a target value of 3.0 INR may be the most appropriate. This target level is considerably lower than intensities used in the SPIRIT trial (3.0 to 4.5 INR) and comes close to the applied intensities (2.0 to $3.0 \mathrm{INR}$ ) in an ongoing trial on the secondary prevention of cerebral ischemia in low-risk patients with cerebral ischemia of presumed arterial origin. ${ }^{20}$

Because we performed an observational study, cardiac sources of embolism might have been present more often in our patients than in the anticoagulated cohort of the randomized trial (SPIRIT). Indeed, 15\% of the pilot sample that we investigated extensively had atrial fibrillation and were consequently misclassified by the anticoagulation clinic. One patient was diagnosed with a thrombus in the heart, but this was looked for neither as inclusion nor as exclusion criterion in the SPIRIT trial.

The participation of patients with stroke of cardiac origin in our study may have led to an underestimation of bleeding incidence in long-term users because cardiac patients have a bleeding risk of only less than $3 \% .^{5}$ It therefore is too soon to postulate that oral anticoagulant therapy for patients with cerebral ischemia of presumed arterial origin is safe for long-term treatment and that the initial high bleeding risk is the only problem to overcome. However, further study of the alarming outcomes of the SPIRIT trial revealed subgroups with a very high bleeding risk. Oral anticoagulant treatment for patients with cerebral ischemia of noncardiac origin is still being debated.

\section{References}

1. Atrial Fibrillation Investigators. Risk factors for stroke and efficacy of antithrombotic therapy in atrial fibrillation. Analysis of pooled data from five randomized controlled. Arch Intern Med 1994;154:1449-1457.

2. The Sixty Plus Reinfarction Study Research Group. A doubleblind trial to assess long-term oral anticoagulant therapy in elderly patients after myocardial infarction. Lancet 1980;2: 989-994

3. Anticoagulants in the Secondary Prevention of Exents in Coronary Thrombosis (ASPECT) Research Group. Effect of longterm oral anticoagulant treatment on mortality and cardiovascular morbidity after myocardial infarction. Lancet 1994;343:499-503.

4. Smith P, Arnesen H, Holme I. The effect of warfarin on mortality and reinfarction after myocardial infarction. $N$ Engl J Med 1990;323:147-152.

5. EAFT (European Atrial Fibrillation Trial) Study Group. Secondary prevention in non-rheumatic atrial fibrillation after transient ischaemic attack or minor stroke. Lancet 1993;342: 1255-1262.

6. The Stroke Prevention in Reversible Ischemia Trial (SPIRIT) Study Group. A randomized trial of anticoagulants versus aspirin after cerebral ischemia of presumed arterial origin. Ann Neurol 1997;42:857-865.

7. Gorter JW for the Stroke Prevention in Reversible Ischemia Trial (SPIRIT) and European Atrial Fibrillation Trial (EAFT) Study Groups. Major bleeding during anticoagulation after cerebral ischemia: patterns and risk factors. Neurology 1999; 53:1319-1327.

8. Rosendaal FR, van der Meer FJM, Cannegieter SC. Management of anticoagulant therapy: the Dutch experience. J Thromb Thrombolysis 1996;2:265-269.

9. International Committee for Standardization in Hematology, International Committee on Thrombosis and Hemostasis. ICSH/ICTH recommendations for reporting prothrombin time in oral anticoagulation control. Thromb Haemost 1982;53: 155-156.

10. Rosendaal FR, Cannegieter SC, van der Meer FJ, Briët E. A method to determine the optimal intensity of oral anticoagulant therapy. Thromb Haemost 1993;69:236-239.

11. Landefeld CS, Goldman L. Major bleeding in outpatients treated with warfarin: incidence and prediction by factors known at the start of outpatient therapy. Am J Med 1989;87: 144-152.

12. The Stroke Prevention in Atrial Fibrillation Investigators. Bleeding during antithrombotic therapy in patients with atrial fibrillation. Arch Intern Med 1996;156:409-416.

13. Rosendaal FR. The Scylla and Charybdis of oral anticoagulant treatment. N Engl J Med 1996;335:587-589.

14. van der Meer FJ, Rosendaal FR, Vandenbroucke JP, Briët E. Bleeding complications in oral anticoagulant therapy. An analysis of risk factors. Arch Intern Med 1993;153:1557-1562.

15. Pantoni L, Garcia JH. Pathogenesis of leukoaraiosis: a review. Stroke 1997;28:652-659.

16. Hart RG, Boop BS, Anderson DC. Oral anticoagulants and intracranial hemorrhage. Facts and hypotheses. Stroke 1995; 26:1471-1477.

17. The European Atrial Fibrillation Trial Study Group. Optimal oral anticoagulant therapy in patients with nonrheumatic atrial fibrillation and recent cerebral ischemia. N Engl J Med 1995;333:5-10.

18. Azar AJ, Cannegieter SC, Deckers JW, et al. Optimal intensity of oral anticoagulant therapy after myocardial infarction. J Am Coll Cardiol 1996;27:1349-1355.

19. Cannegieter SC, Rosendaal FR, Wintzen AR, van der Meer FJ, Vandenbroucke JP, Briët E. Optimal oral anticoagulant therapy in patients with mechanical heart valves. $N$ Engl J Med 1995;333:11-17.

20. de Schryver ELLM on behalf of the European/Australian Stroke Prevention in Reversible Ischaemia Trial (ESPRIT) group. Design of ESPRIT: an international randomized trial for secondary prevention after non-disabling cerebral ischaemia of arterial origin. Cerebrovasc Dis 2000;10:147-150. 